\title{
COMMENTAIRE DE RENAULT
}

\section{Éric Vaillant}

Le rapport "Métallurgie, science et ingénierie 》 est un document de référence pour l'ensemble des acteurs de cette filière. Il met en évidence un présent encore assez brillant tout en alertant sur un avenir proche inquiétant quant à la pérennité et au développement de ce secteur clé de l'industrie française.

Il faut sans doute pondérer certaines parties du rapport qui mettrait en compétition les matériaux métalliques avec d'autres solutions (thermoplastiques, composites ,...) car la compétitivité de certaines activités emblématiques de l'industrie française (transport, énergie, ...) repose en partie sur la diversité des choix technologiques matières proposés.

Ce rapport au travers des différentes thématiques abordées montre la nécessité d'inventer le concept de " métallurgie durable».

L'essentiel des leviers de compétitivité de la filière est mis en évidence dans le document, je souhaiterais cependant mettre l'accent sur six points à développer ou renforcer en lien avec les besoins de l'industrie automobile :

- la corrosion, point faible des matériaux métalliques est un domaine dans lequel il y a un besoin de développement de compétences par la communauté scientifique pour garder des matériaux compétitifs d'autant que les dimensions « comestique » (état neuf) et « vieillissement » d'un produit sont aujourd'hui des facteurs décisifs dans le choix des consommateurs. La corrosion automobile $n^{\prime}$ est pas traitée de manière spécifique par les laboratoires et universités (contrairement à d'autres industries telles que le nucléaire, la pétrochimie ou encore le naval) alors qu'elle présente des spécificités : les fondants routiers, l'écoulement des polluants dans et autour du véhicule,...

- la sidérurgie française si elle se doit d'être une vitrine technologique par sa capacité à proposer des alliages spéciaux doit aussi montrer sa faculté à accompagner ses clients dans le développement de produit « low cost ", pour lesquels des matériaux à bas coût sont un levier majeur de la performance (voir Logan pour Renault). La capacité des sidérurgistes à maîtriser la dispersion/répétabilité des produits (travaux à impulser par les laboratoires universitaires) doit permettre de " décranter » le niveau de gamme de ceux-ci pour certaines applications; 
- le management des incertitudes doit devenir une priorité de la filière. En effet la croissance passe par une meilleure capacité "d'accommodation » au caractère évolutif de la réglementation (voir norme REACH mais aussi CAFE pour la dépollution automobile) ainsi qu'à la prise en compte de la rareté des matières premières. Or, celle-ci est un facteur limitant sévère face auquel l'Europe est particulièrement vulnérable. L'effort fait sur la recyclabilité des métaux (et plus encore sur la conception de produits permettant un recyclage facile) est nécessaire, il doit aussi être accompagné d'une mobilisation quant à la capacité à innover pour proposer des alternatives à ces matériaux rares et donc chers;

- la compétitivité des matériaux proposés sera jugée à l'aune de l'analyse du cycle de vie de ceux-ci, intégrant non seulement les dépenses énergétiques engagées (dimension globalement bien intégrée par les fabricants) mais aussi les ressources hydriques " or bleu » afin de libérer la filière d'un comportement " aqua addicted " non durable. L'analyse du cycle de vie va tirer les solutions techniques de l'obtention à l'utilisation, d'où la nécessité de remise en cause des process usités jusqu'alors ;

- l'allègement, préoccupation majeure de l'industrie automobile sous contrainte de limitation des émissions de $\mathrm{CO}_{2}$ donc de réduction de la consommation, pousse les constructeurs automobiles à rechercher des matériaux ayant un rapport masse/performance optimisé. Pour certaines applications cela passe nécessairement par des solutions innovantes et performantes (durabilité, usure) dans le domaine des « surfaces et interfaces » afin de réduire l'épaisseur du substrat tout en préservant les caractéristiques fonctionnelles. Le développement des revêtements couche mince (tels que les DLC) est un bon exemple du besoin dans ce domaine, dont l'expansion nécessite une implication forte des industriels et chercheurs;

- les techniques d'assemblage ont énormément progressé ces dernières années tant dans leur capacité à assurer le comportement mécanique d'une pièce que dans la mise en œuvre industrielle. Le contrôle de ces assemblages en chaîne de fabrication reste le maillon faible de ces techniques, limitant ainsi parfois leur compétitivité (temps de cycle limité par le prélèvement pour contrôle destructif ou besoin de redondance dans la liaison). Les techniques de contrôle non destructif des assemblages, si elles sont nombreuses et très utilisées dans les phases de développement des produits en laboratoire, doivent encore progresser dans leur mise en œuvre en contexte industriel de grande cadence. Un effort particulier doit être fait par les chercheurs dans ce domaine connexe à la métallurgie pour proposer des solutions techniques accompagnant le développement des nouveaux matériaux et les process d'assemblage associés. 